$\begin{array}{cl}\begin{array}{c}\text { Revue } \\ \text { de /histoire }\end{array} & \text { Revue de l'histoire des religions } \\ \text { des religions } & \begin{array}{l}3 \mid 2012 \\ \text { Varia }\end{array}\end{array}$

\title{
Mos orandi. La législation des usages liturgiques au Moyen Âge (XII ${ }^{\mathrm{e}}-\mathrm{XV}^{\mathrm{e}} \mathrm{s}$.)
}

Mos orandi. The Legislation of Liturgical Uses in the Middle Ages (1100-1500)

Jean-Baptiste Lebigue

\section{OpenEdition}

Journals

Édition électronique

URL : http://journals.openedition.org/rhr/7906

DOI : $10.4000 /$ rhr.7906

ISSN : 2105-2573

Éditeur

Armand Colin

Édition imprimée

Date de publication : 1 septembre 2012

Pagination : 349-373

ISBN : 978-2200-92975-0

ISSN : 0035-1423

Référence électronique

Jean-Baptiste Lebigue, " Mos orandi. La législation des usages liturgiques au Moyen Âge (XIle-XVe s.) », Revue de l'histoire des religions [En ligne], 3 | 2012, mis en ligne le 01 septembre 2015, consulté le 04 mai 2019. URL : http://journals.openedition.org/rhr/7906 ; DOI : 10.4000/rhr.7906 


\section{Mos orandi \\ La législation des usages liturgiques au Moyen Âge $\left(\mathrm{XII}^{\mathrm{e}}-\mathrm{XV}^{\mathrm{e}} \mathbf{S}\right.$.)}

Du XII siècle au concile de Trente, les églises locales et les ordres réguliers fixèrent avec de plus en plus de précision les usages liturgiques qui leur étaient propres. Ordinaires et bréviaires, où elles firent la synthèse des prescriptions relatives au service divin, leur servirent à promulguer, enregistrer et diffuser ces coutumes cultuelles en constante évolution. Plus que les recueils de statuts capitulaires et synodaux, ces livres peuvent être considérés comme de véritables codes juridiques des usages. La Réforme catholique et la fondation de la Sacrée Congrégation des Rites en 1588 firent peu à peu disparaître cette faculté des églises locales, en particulier des chapitres de cathédrales, d'édicter la législation de leur liturgie.

\section{Mos orandi. The Legislation of Liturgical Uses in the Middle Ages (1100-1500)}

From the $12^{\text {th }}$ century until the Council of Trent, local churches and regular orders determined their own liturgical uses with ever greater precision. They used ordinals and breviaries, which synthesized the prescriptions concerning the divine service, to promulgate, record and spread these continually evolving cultual customs. These books may be considered as the true legal codes of their uses, more than as simply the collections of synodal or capitular statutes. As a result of the Catholic Reformation and the foundation of the Roman Congregation of Rites in 1588, local churches, particularly cathedral chapters, were less and less entitled to their own liturgical rules. 
L'historien en quête des pratiques cultuelles du Moyen Âge est tôt ou tard confronté à la notion d'usage liturgique. Un bréviaire à l'usage de Paris diffère d'un autre à l'usage de Rouen, mais aussi d'un bréviaire romain, cistercien ou chartreux ${ }^{1}$. Usages multiples, qui font varier, dans une même ville, la liturgie d'une église à l'autre. Les offices célébrés dans une cathédrale, qu'on retrouve sous une forme allégée dans les paroissiales du diocèse, ne sont pas identiques dans les collégiales, abbayes et couvents du lieu, que ces derniers soient ou non soumis à la juridiction de l'évêque. Leurs pratiques cultuelles peuvent s'associer, par exemple lors de processions collectives ou de stations dans les églises voisines; se ressembler, par l'institution simultanée de nouvelles fêtes ou par une dévotion commune à des saints locaux; mais elles ne coïncident pas.

Désignés dans les livres liturgiques sous le terme d'usus, consuetudines, mores, parfois ritus, ces usages fortement identitaires se fixèrent avec une précision grandissante à partir $\mathrm{du} \mathrm{XII}^{\mathrm{e}}$ siècle, sans pour autant cesser d'évoluer ensuite. C'est pourquoi ils constituèrent une part importante de la législation des églises, quoique leurs traces soient peu nombreuses dans les statuts capitulaires ou synodaux. C'est plutôt dans ces recueils très élaborés auxquels on donne le nom d'ordinaires, où les spécificités cultuelles d'une église sont décrites pour chaque jour de l'année,

1. Pour la nomenclature des livres liturgiques, le lecteur pourra se reporter à l'Initiation aux manuscrits liturgiques, Paris, IRHT, 2007 (Ædilis, Publications pédagogiques, 6). En ligne: http://cel.archives-ouvertes.fr/cel-00194063. Quelques termes indispensables méritent néanmoins d'être précisés ici :

Pièce: tout chant, lecture ou oraison dits ou chantés lors d'une cérémonie liturgique, qu'ils soient copiés in extenso ou seulement désignés par leurs incipit.

Propre: ensemble des pièces propres à l'office et à la messe d'une fête, d'un dimanche ou d'une férie donnés. On appelle «temporal» l'ensemble des propres du temps (voir infra, note 4); «sanctoral», ceux des fêtes de saints.

Ordo (pluriel: ordines) : terme générique désignant l'ensemble des prescriptions relatives à une ou plusieurs cérémonies. Peuvent y être indiqués - non inclusivement - le choix et l'ordre des pièces, la manière de les dire, la place, les ustensiles, les acteurs et le déroulement des cérémonies, le moment ou les périodes de l'année où elles doivent avoir lieu.

Article: unité textuelle regroupant prescriptions et pièces afférentes à un office, une messe ou une cérémonie donnés. L'article peut correspondre à un propre, à une section du commun des saints ou à un ordo. 
qu'il en faut chercher le témoignage. Produits et promulgués par les chapitres et synodes, les ordinaires doivent être considérés comme de véritables codes coutumiers des usages liturgiques. Plus que les canons, statuts et ordonnances touchant à la discipline du clergé ou à l'administration des sacrements, souvent stéréotypés, ils rendent compte de la faculté des églises locales d'édicter un droit ecclésiastique particulier. L'exercice de cette puissance législative, encore vivace à la fin du Moyen Âge, s'étiola brutalement avec l'entreprise d'unification liturgique issue du concile de Trente, relayée par les décrets de la Sacrée Congrégation des Rites, que Sixte Quint fonda en 1588.

\section{1/ IDENTIFICATION ET TENEUR DES USAGES LitURGIQUES}

Grâce aux travaux des Bollandistes, de Grotefend, Dom Bessac, Victor Leroquais et, récemment, au Calendoscope de Denis Muzerelle, il est devenu aisé d'identifier l'usage de la plupart des manuscrits liturgiques, même en l'absence de sa mention explicite dans le volume. Il suffit généralement de relever les fêtes portées au calendrier ou pourvues d'un propre dans le sanctoral, les saints locaux invoqués dans les litanies. Certains livres, tels les pontificaux, manuels et autres recueils d'ordines réclament une enquête plus minutieuse, dont Victor Leroquais a exposé les principes en introduction de son Catalogue des pontificaux manuscrits des bibliothèques publiques de France ${ }^{2}$. Ces méthodes, toujours employées, ont fait les preuves de leur efficacité. Leur seul tort est de masquer la teneur véritable des usages, dont la nature ne se réduit pas à une simple liste de fêtes caractéristiques ou à des indices glanés au fil des rubriques. Les variations qui font différer les usages médiévaux sont d'une tout autre ampleur que l'ajout de «propres» en annexe des bréviaires ou des missels de la fin du $\mathrm{XIX}^{\mathrm{e}}$ siècle ${ }^{3}$. Elles tiennent non seulement au calendrier festif, au

2. Victor Leroquais, Catalogue des pontificaux manuscrits des bibliothèques publiques de France, Mâcon, Protat, 1940-1941, 3 vol., en part., t. 1, p. C-CVI.

3. Dans ce dernier cas, le «propre» d'une nation, d'un diocèse ou d'un ordre religieux se limite à l'adjonction d'offices ou de messes pour quelques fêtes locales dont la Sacrée Congrégation des Rites a autorisé la célébration, mais sans que le corps du bréviaire ou du missel ainsi supplémenté ne soit affecté dans le reste de sa composition. L'une des difficultés majeures de l'étude de la liturgie provient de 
culte rendu à des saints particuliers, à des cérémonies que leur lieu et leur date rendent distinctives (processions, synodes, chapitres, anniversaires de défunts, de dédicaces, d'inventions, réceptions ou translations de reliques, etc.), mais aussi au choix ou à la création de chants, lectures ou oraisons dans les propres du temps et des saints, à une échelle d'appréciation des offices et à la manière de résoudre les cas où les divers cycles annuels les amènent à se superposer ${ }^{4}$. Ces trois derniers aspects, souvent négligés, doivent être expliqués, si l'on veut saisir la nécessité ressentie par les églises médiévales d'édicter et d'organiser leurs coutumes liturgiques.

\section{1. Fixation et évolution des propres}

L'office d'une même fête ou d'un même dimanche n'est pas forcément semblable selon l'usage dont il est issu. Le phénomène, qui touche jusqu' aux fêtes universelles comme Noël ou l'Ascension, est mieux connu pour les chants propres de la messe et de l'office, grâce aux indispensables répertoires de Dom Hesbert, qui a isolé les traditions les plus anciennes sans pour autant épuiser la matière 5 . Les grands corps d'édition de formules euchologiques (oraisons, préfaces, bénédictions, etc.) nous ouvrent à la richesse du fonds

la polysémie des termes usuels. La notion de «propriété» peut à la fois s'appliquer aux particularismes cultuels d'une église donnée, et à désigner chants, oraisons ou lectures spécifiques d'un dimanche, d'une férie ou d'une fête. C'est dans ce dernier sens qu'il est désormais employé dans le présent article.

4. On compte trois cycles annuels fondamentaux : celui du temps, qui comprend le cycle hebdomadaire des dimanches et féries, les fêtes et temps liturgiques axés sur Pâques (Septuagésime, Carême, temps pascal, Pentecôte, Fête-Dieu, etc.) et Noël (de l'Avent à l'octave de l'Épiphanie) - celui des saints, auquel se rattachent les fêtes fixes de l'année hors temps de Noël, y compris les dédicaces et autres anniversaires solennels - et celui des morts (anniversaires des défunts, obits et fondations). D'autres cycles viennent s'enter sur les précédents, sans les recouper exactement: programme des lectures bibliques à matines et au réfectoire sur l'année (historia des dimanches), alternances entre été et hiver (par exemple pour l'hymne de matines), choix des messes et offices votifs, ou des antiennes mariales à complies, variations dans les offices surérogatoires tels les heures de la Vierge, etc.

5. René-Jean Hesbert, Antiphonale missarum sextuplex, Bruxelles, Vromant, 1935; Idem, Corpus antiphonalium officii, Rome, Herder, 1963-1979. Ce corpus déjà considérable ne représente qu'une petite portion des chants ecclésiastiques catalogués par les contributeurs de la base Cantus (en ligne: http://publish.uwo. $\mathrm{ca} / \sim$ cantus/). 
textuel médiéval ${ }^{6}$; mais ils sont impuissants à manifester l'infinie variété des combinaisons selon les usages, le croisement ou la contamination des traditions, leur enrichissement ininterrompu au fil des siècles ${ }^{7}$. Quant aux leçons de matines, hormis le programme de l'historia biblique et les séries de péricopes homilétiques tirés des Pères, le reste demeure un vaste champ à explorer: passages de Vies de saints et de miracles de toute origine, textes et sermons d'auteurs médiévaux parfois tardifs, extraits de chroniques ou de canons conciliaires, composition originales résumant des légendes hagiographiques, etc.

La part des créations textuelles et musicales n'est pas négligeable dans l'évolution des usages ${ }^{8}$. Sans même parler des canonisations de saints, de l'invention ou du transfert de reliques, entraînant la composition de nouveaux offices, des fêtes d'institution antique furent parfois pourvues de nouveaux chants, lectures et oraisons par les églises locales ${ }^{9}$. De même, la multiplication des tropes, proses et séquences, extrapolant les chants du propre et du commun, de la messe et de l'office, a considérablement accentué la physionomie particulière de leurs liturgies respectives. À quoi s'ajoute encore la diversité des pratiques du chant, ardue à mesurer par les seules notations musicales dans les manuscrits, mais dont témoignent la spécialisation cantorale de certains chapelains et vicaires, comme les célèbres machicots de Notre-Dame de Paris, et la constitution de maîtrises dans les cathédrales dans les derniers siècles du Moyen $\hat{\text { Agge }}{ }^{10}$.

6. Voir l'édition typologique de ces ensembles de sources dans la collection du Corpus Christianorum Series latina: Corpus orationum (t. 160-160/H), Corpus praefationum (t. 161-161/D) et Corpus benedictionum pontificalium (t. 162-162/C).

7. On relève des variations analogues dans les ordines rituels, en particulier ceux contenus dans les pontificaux.

8. L'activité créatrice n'a pas tari à la fin du Moyen Âge, comme en témoigne la riche moisson d'hymnes, séquences et offices rythmiques de Clemens Blume et Guido Maria Dreves dans les Analecta hymnica, Leipzig, 1888-1922, réimpr. New York, Londres, Johnson reprint Corporation, 1961, 55 t.

9. Voir par exemple la thèse de Jean-François Goudesenne, Les offices ou historiae composés pour les fêtes de saints dans la province ecclésiastique de Reims, 775-1130, Turnhout, Brepols, 2002. Beaucoup de découvertes restent à faire pour la période postérieure au XII ${ }^{\mathrm{e}}$ siècle.

10. On distingue ici la schola cantorum, attestée dans les cathédrales depuis le Haut Moyen Âge, des maîtrises, psallettes et autres manécanteries, qui commencent à s'établir au plus tard dans les premières décennies du XIII ${ }^{\mathrm{e}}$ siècle. $\mathrm{Si}$ la filiation entre ces deux types d'institutions est indéniable, les maîtrises supposent 
Les répertoires spécifiques à chaque église ne se sont stabilisés que progressivement. $\mathrm{Au} \mathrm{X}^{\mathrm{e}}$ siècle encore, il est fréquent de trouver des pièces surnuméraires dans les propres de certains dimanches ou fêtes: collectes, versets de l'antienne d'offertoire, répons ou leçons de matines, etc., sans que leur affectation à telle férie, octave ou mémoire soit précisée. Il semble qu'on ait puisé longtemps dans un fonds assez large de pièces liturgiques, peu à peu réduit, ordonné et figé. Au milieu du XIII ${ }^{\mathrm{e}}$ siècle, la plupart des flottements dans l'attribution des pièces a disparu. L'une des causes de cette précision croissante est la composition des missels et bréviaires, livres synthétiques assemblant pour chaque propre tous les chants, lectures et oraisons nécessaires à la messe et à l'office. Le phénomène est flagrant dans les bréviaires, même quand ils sont répartis en plusieurs volumes: le processus de concentration et de sélection a provoqué l'appauvrissement du répertoire et l'abréviation des pièces les plus encombrantes, surtout les lectures, tant leçons de matines que capitules. Tout élément surnuméraire ou sans affectation explicite n'y pouvait subsister. Les raisons qui ont mené à la rédaction des missels et bréviaires ne sont pas seulement pratiques. Certes, ces nouveaux livres sont particulièrement adaptés au développement des messes privées et à la récitation individuelle des heures canoniales, contrepartie d'un lent déclin des offices communautaires. Cette décadence présumée de la liturgie chorale, souvent invoquée, est contredite par l'activité de nombreux chapitres de cathédrales ou de collégiales aux XIII et XIV ${ }^{\mathrm{e}}$ siècles, époque d'intense réglementation des devoirs du clergé au chœur, de multiplication et de spécialisation poussée des acteurs du culte. L'argument d'une pratique individuelle, qui pourrait n'être que la projection anachronique d'habitudes modernes, me semble moins décisif que le désir, avoué par les sacristes médiévaux, d'obtenir une meilleure maîtrise des offices ecclésiastiques, en vue d'en réduire

une spécialisation cantorale des enfants, probablement due à l'élargissement du répertoire polyphonique, et leur subordination, avec d'autres chanteurs, adultes et chevronnés, à un maître de chapelle (magister cantus), distinct du chantre et du préchantre. En attendant une étude d'ensemble sur le sujet, on se reportera aux monographies les plus récentes sur des cas d'espèces, comme celle consacrée à la cathédrale parisienne par Craig Wright, Music and Ceremony at Notre Dame of Paris, 500-1550, Cambridge, Cambridge University Press, 1989, en part. p. 165195. 
les ambiguïtés, causes de conflits au sein des communautés ${ }^{11}$. La constitution des missels et bréviaires témoigne ainsi d'un mouvement profond de mise en cohérence interne de chaque usage, préparant leur synthèse ultime dans l'élaboration d'un ordinaire.

\section{2. Échelles d'appréciation des offices}

Cette tendance est aussi sensible dans le perfectionnement d'échelles d'appréciation des offices, qui apparaissent au cours $\mathrm{du} \mathrm{XII}^{\mathrm{e}}$ siècle dans les calendriers ecclésiastiques: les cérémonies y sont caractérisées de plus en plus précisément par des mentions de «rits». Il s'agit de critères renvoyant à des pratiques cultuelles ou cantorales distinctives, et dont l'expression, nécessairement succincte, est apposée à l'intitulé de la fête. Pour n'en donner qu'un exemple, voici la séquence des offices tombant du 7 au 11 août dans le calendrier placé en tête d'un bréviaire à l'usage de la cathédrale de Cambrai, tous marqués par un rit différent (signalé ici par des caractères gras) $^{12}$ :

7 août - Donati episcopi et martyris, III lectiones.

8 août - Cyriaci martyris, III lectiones cum memoria.

9 août - Romani militis, III responsoria ; vigilia [s. Laurentii], III lectiones, memoria.

10 août - Laurentii martyris, Ix lectiones, magnum duplum.

11 août - Gaugerici episcopi et confessoris, IX lectiones, dimidium duplum.

Certains de ces critères sont universels dans l'Église latine, telle la distinction fondée sur le nombre de leçons à matines entre offices de type férial (III lectiones) et dominical (IX lectiones), qui influent sur le choix des psaumes et sur la structure même des offices. Les seconds, plus solennels, comptent trois nocturnes à matines et deux offices de vêpres ${ }^{13}$, comme un dimanche, au lieu d'un seul nocturne et une seule heure de vêpres pour le type férial, c'est-à-dire le modèle

11. La plupart des prologues d'ordinaires évoque en effet les dissentiones, scandala, altercationes, contentiones, scismata, etc., naissant de traditions cultuelles incertaines ou équivoques.

12. Cambrai, BM, ms. 33, f. 5. Cambrai, $3^{\mathrm{e}}$ quart du XIII ${ }^{\mathrm{e}}$ siècle (après 1253).

13. Premières vêpres la veille de la fête, et secondes vêpres le jour même. 
suivi pour les jours de semaine (féries). Très répandus aussi dans les divers usages sont les rits semi-double (semiduplex, semiduplum, cum semiduplo, dimidium duplum) et double (duplex, duplum), avec tout un éventail de variantes: tiers-double (tertium duplum), grand double (magnum duplum), tout double (totum duplum), triple (triplex), quadruple (quadruplex), etc. Cette impression d'uniformité relative est trompeuse. Selon l'époque ou le lieu, ces mentions sont entendues différemment, s'appliquant soit au doublement partiel ou complet des antiennes, soit à la célébration de deux offices de vêpres le même jour, soit au nombre d'exécutants requis pour certaines pièces de chant. Plus changeante encore selon les usages est la terminologie se rapportant à l'étendue des propres, c'està-dire au nombre de lectures, chants ou oraisons appropriés à un office particulier. Dans l'exemple cambrésien cité plus haut, cette distinction concerne plus spécialement les rits III responsoria, III lectiones et III lectiones cum memoria. On relève une infinie variété d'appellations fondées sur le même principe: memoria, III lectiones et III lectiones sollempnes à Saint-Victor de Paris, ou encore fit, fit cum propria oratione, III lectiones et 'Te Deum' dans la cathédrale d'Amiens ${ }^{14}$. D'autres rits différencient la solennité d'un office par le luminaire (nombre de cierges) ou, souvent en milieu monastique, par le port d'ornements liturgiques. Aux fêtes in albis, le chœur est vêtu d'aubes comme à Pâques et durant son octave; à celles in capis, on officie en chapes, elles-mêmes objet d'appréciation par le nombre ou la qualité: in tribus capis, in sex capis, in parvis capis, in magnis capis, in duplicibus capis (en chapes pour offices doubles), etc. C'est à partir de la fin du XIII ${ }^{\mathrm{e}}$ siècle que les églises poussèrent le plus loin l'inventivité, contraintes par l'adoption cumulative de nouvelles fêtes à en affiner la hiérarchie, créant à leur seul usage des expressions défiant l'interprétation, voire la syntaxe et le bon sens. De cette époque datent les rits amiénois magnum duplum sine 'cum eo', et magnum duplum et 'cum eo'; ou encore le $P$. C. (in paratis capis) et le F. Pro. D. C. (festum processionaliter [sic] in duplicibus capis) de l'abbaye Saint-Nicolas d'Angers ${ }^{15}$.

14. Tables de comput et calendrier à l'usage de Saint-Victor de Paris, peu après 1403 (Paris, Bibl. de l'Institut de France, ms. 4); Ordinaire à l'usage de la cathédrale d'Amiens, de 1291 (Amiens, BM, ms. 184).

15. Amiens, voir ibid.; Ordinaire à l'usage de l'abbaye Saint-Nicolas d'Angers, de la seconde moitié du xv $v^{\mathrm{e}}$ siècle (Angers, BM, ms. 89). 


\subsection{Règles de préséances entre les offices}

Ces particularismes regardant l'échelle d'appréciation des offices resteraient anecdotiques s'ils ne conditionnaient la façon de résoudre les cas de superposition entre les propres. Ce type de problèmes surgit par exemple en situation d'occurrence, quand une fête de saint tombe le même jour qu'un dimanche, une férie privilégiée ou une fête mobile. Lequel des deux offices doit-il prendre le pas sur l'autre? Que faire de l'office jugé inférieur? L'omettre, en faire mémoire le jour même, le transférer en entier ou partiellement la veille ou le lendemain, panacher les deux propres? Et avec quelles conséquences pour les fêtes adjacentes? De même, quel parti prendre dans un cas de concurrence, c'est-à-dire lorsque les secondes vêpres d'un office de type dominical rencontrent les premières vêpres de l'office marqué au lendemain? Or les cycles liturgiques, qui combinent anniversaires fixes, fêtes mobiles et rythme hebdomadaire, se recoupent différemment d'une année sur l'autre, multipliant à l'infini les occasions d'interférences entre les propres.

Les règles qui permettent de surmonter de telles difficultés dépendent étroitement des rits assignés aux offices, et chaque église possède sa méthode pour remédier aux incessantes coïncidences. Cette part considérable des usages reste impalpable dans la plupart des livres cultuels: sacramentaires, graduels, antiphonaires, processionnaux, collectaires ou lectionnaires sont conçus pour compiler les pièces (oraisons, chants, lectures) nécessaires à la récitation des divers offices, non pour en prévoir l'agencement accidentel, soumis aux aléas du calendrier. À partir du milieu du XIII ${ }^{\mathrm{e}}$ siècle, certains bréviaires, collectaires et missels commencent toutefois à fournir des indications à ce sujet. Celles-ci sont limitées par la taille forcément réduite des rubriques, à l'exception des «tables de l'Avent», généralement copiées entre le calendrier et le début du temporal ${ }^{16}$. Il s'agit de textes de quelques pages décrivant, selon le jour de la semaine où tombe le 25 décembre, l'organisation des quatre semaines précédant Noël, avec toutes les combinaisons

16. On peut également trouver d'importants corps de prescriptions guidant la célébration des fêtes pendant certains temps liturgiques comme la Septuagésime, le Carême et le temps pascal. 
possibles entre le propre du temps et des saints ${ }^{17}$. Ces tables de l'Avent, qui ne règlent les questions de préséances entre les offices que pour une courte période de l'année, proviennent des ordinaires. Ceux-ci rendent les mêmes services pour la totalité du cycle annuel. L'article-type d'un ordinaire est conçu de la façon suivante: après avoir cité les incipit des pièces assignées à l'office, apporté les précisions sur les exécutants, le luminaire, les sonneries de cloches, le vestiaire et le mobilier requis pour les cérémonies, puis détaillé le déroulement de la journée, le parcours des processions, le montant des distributions, etc., sont résolus les cas les plus épineux de concurrences et d'occurrences pouvant interférer avec la liturgie du jour. L'un des plus complets à cet égard est l'exemplaire composé sous la direction de Raoul de Rouvroy à l'usage de la cathédrale d'Amiens en $1291^{18}$. La maîtrise du calendrier liturgique y est stupéfiante, puisque le chanoine d'Amiens a su, pour toute occasion, prévoir l'incidence des cycles de lectures de matines, distribuer si nécessaire les pièces propres des offices empêchés en les reportant à d'autres jours libres, et analyser jusqu'aux «réactions en chaîne » provoquées par ces transferts influant sur les offices voisins ${ }^{19}$. Cette œuvre témoigne de la complexité inouïe des usages liturgiques, mais aussi de la remarquable cohérence que surent leur donner les sacristes à la fin du Moyen Âge.

\section{2/ Organes et MOdalités de la PROMUlgation des USAGES LITURGIQUES}

Les usages liturgiques n'auraient pu parvenir à un tel degré de subtilité et de contrainte sans l'assise institutionnelle des assemblées et autorités édictant le droit ecclésiastique: chapitres de cathédrales, collégiales et abbayes, synodes, chapitres généraux

17. Le temps de l'Avent provoque des cas fort complexes de superposition des propres, entre dimanches et féries privilégiés et des fêtes aussi importantes que la Saint-André, la Saint-Nicolas ou la Sainte-Lucie.

18. Amiens, BM, ms. 184, édité par Georges Durand, Ordinaire de l'église Notre-Dame, cathédrale d'Amiens, Amiens-Paris, 1934 (Mémoires de la Société des antiquaires de Picardie, 22).

19. Voir l'exemple commenté de la fête de saint Nicaise à la cathédrale d'Amiens, dans l'Initiation aux manuscrits liturgiques, op. cit., au chapitre intitulé «Règles de préséances entre les offices ». 
des ordres réguliers, conciles provinciaux et généraux, sans oublier la papauté. Or l'historiographie de la fin du Moyen Âge fausse notre compréhension de l'activité législative des organes locaux en matière cultuelle: l'institution par les papes de fêtes qu'ils destinaient à devenir universelles dans l'Église latine, telles la Saint-François ou la Fête-Dieu, a rejeté dans l'ombre l'évolution des usages à la même époque et leur fondement juridique propre. De fait, l'illusion d'un droit strictement hiérarchique, où l'autorité papale entraînerait automatiquement l'adoption des offices qu'elle promulgue par des «instances inférieures», est un anachronisme qui ne résiste pas à l'examen. L'enquête que Théophile Desbonnets mena dans les bréviaires catalogués par Victor Leroquais pour compiler les propres de la Saint-François est révélatrice ${ }^{20}$. Rares sont les cas où l'office du saint d'Assise répond aux exigences exprimées par Grégoire IX dans la bulle Mira circa nos du 19 juillet $1228^{21}$. $\mathrm{Au}$ lieu de l'office solennel qui devait être célébré le 4 octobre par l'Église universelle, on trouve majoritairement un simple office de type férial, le plus souvent dépourvu de propre, quand il n'est pas purement absent du calendrier festif. Les lenteurs qui échelonnèrent l'adoption de la Fête-Dieu (Corpus Christi) par les divers usages sur plus d'un demi-siècle, pourtant en pleine flambée de la dévotion eucharistique, peuvent être interprétées dans le même sens. La promulgation d'Urbain IV en 1264 obtint peu de succès; celle de 1311 au concile de Vienne en eut davantage; mais son application dut tout de même attendre le relais des conciles provinciaux, dont les décrets s'insérèrent dans les collections synodales des diocèses. Au mieux, l'usage de la curie romaine était considéré comme un modèle vénérable dont il était bienvenu de s'inspirer, mais pas de nature à se substituer aux traditions particulières sans la sanction des organes ecclésiastiques locaux.

20. Théophile Desbonnets, «La diffusion du culte de saint François en France d'après les bréviaires manuscrits étrangers à l'Ordre», dans Archivum franciscanum historicum, t. 75 (1982), p. 153-215.

21. Voir Olivier Legendre, «Grégoire IX. Bulle de canonisation de François d'Assise», dans François d'Assise. Écrits, Vies, témoignages, éd. Jacques Dalarun, Paris, Éditions franciscaines, Cerf, 2010, t. 1, p. 414-427. 


\subsection{Législation cultuelle des églises locales}

Contrairement aux décisions papales, bien documentées, les ordonnances des églises en matière liturgique n'apparaissent souvent qu'à travers des témoignages indirects. Les collections de statuts aussi complètes et organisées que celles du chapitre général de l'ordre cistercien font exception au Moyen Âge ${ }^{22}$. Quant aux recueils de délibérations capitulaires des cathédrales et collégiales, il s'agit généralement de sélections raisonnées, en vue de compiler en un seul volume les coutumes en vigueur pour la discipline à l'intérieur du chœur et du cloître, les obligations de chacun, qu'elles soient cultuelles ou non, le versement des distributions, amendes et redevances, mais fort rarement de statuts liturgiques comme l'institution d'une fête ou la modification d'un office. Cette omission est assez compréhensible: ces aménagements ponctuels, peu significatifs quand ils sont énoncés isolément, sont entérinés par la copie de nouveaux livres liturgiques ou, à défaut, par la supplémentation des anciens sous forme d'annotations marginales ou de feuillets insérés. Enfin et surtout, il est beaucoup plus intéressant de les intégrer aux ordinaires pour les remettre en situation dans le cycle liturgique annuel, et les relier ainsi aux autres pratiques constitutives de l'usage ${ }^{23}$.

Certains ordres, tels les cisterciens ou les guillelmites, ont produit des ordinaires munis de renvois systématiques à leurs coutumiers et à leurs statuts ${ }^{24}$. Ces documents, qui supposent à

22. Voir l'édition en huit volumes des statuts cisterciens par Joseph-Marie Canivez, Statuta capitulorum generalium ordinis Cisterciensis ab anno 1116 ad annum 1786, Louvain, 1933-1941. Sur les origines et les premiers textes de la législation cistercienne, voir les nombreuses études dues à Chrysogonus Waddel.

23. Il est malaisé de fournir une évaluation du nombre d'ordinaires conservés aujourd'hui, et à plus forte raison de ceux produits au Moyen Âge. Pour s'en tenir aux fonds des bibliothèques publiques de France, l'ordre de grandeur est de quelque deux cent cinquante ordinaires «stricts», auxquels s'ajoute une proportion au moins équivalente de coutumiers liturgiques et textes normatifs apparentés. C'est peu; mais le taux de destruction dut être considérable, pour les raisons que j'ai avancées en traitant des «Chapelles livresques et ministres désignés: les véritables destinataires des manuscrits de la liturgie pontificale», dans Gazette du livre médiéval, t. 54 (2009), p. 19-30, en part. p. 20-23.

24. Voir J.-B. Lebigue, «Les cérémoniaux manuscrits à la fin du Moyen Âge», dans Les cérémoniaux catholiques en France à l'époque moderne. Une littérature de codification des rites liturgiques, éd. Cécile Davy-Rigaud, Bernard Dompnier et Daniel-Odon Hurel, Turnhout, Brepols, 2009, p. 45-58, en part. p. 55 et $57-58$. 
proximité la présence de vastes collections de textes normatifs, sortent de l'habitude. Dans l'écrasante majorité des cas, l'économie de la rédaction empêche de conserver du décret d'origine autre chose que les prescriptions indispensables à l'accomplissement du culte. Néanmoins, il arrive que les rectificatifs les plus récents, donc les plus susceptibles de contestation, gardent trace du statut primitif. Son souvenir affleure parfois dans une simple date accolée à l'intitulé d'un office ${ }^{25}$, mais peut laisser une empreinte plus sensible. C'est le cas par exemple pour la fête de la conception de la Vierge (8 décembre) dans l'ordinaire-coutumier à l'usage de Saint-Germain-des-Prés, composé sous la direction de son abbé Guillaume L'Évêque en 1395:

La fête de la conception de la bienheureuse Vierge Marie était, d'antiquité, une fête double. Mais parce qu'au temps du seigneur Guillaume abbé de ce lieu, il y eut une grande controverse entre la vénérable université de Paris et les frères prêcheurs ou jacobins, qui erraient gravement au sujet de ladite conception de la bienheureuse Vierge, insultaient ou attaquaient la glorieuse Vierge dans ceux qui lui étaient dévoués; et que, pour cette raison, beaucoup de ces mêmes frères, maltraités, révoquèrent publiquement leurs erreurs et, mis en prison après avoir été copieusement vilipendés en conséquence, furent exclus par la susdite université; c'est pourquoi le susdit abbé Guillaume, troisième du nom, mu par sa dévotion à la louange et à la gloire de la bienheureuse Vierge, décida et ordonna de célébrer cette fête «en petites chapes» et d'y faire en toute chose ce qu'il est coutume de faire pour de telles fêtes ${ }^{26}$.

25. Par exemple à la fête célébrant la translation de la sainte Couronne, qui eut lieu en 1239, est accolée la mention de l'année 1242, datant son institution dans trois ordinaires à l'usage des trinitaires, du milieu du XIII' siècle, voir J.-B. Lebigue, «Le modèle liturgique de Saint-Victor de Paris et son adoption par l'ordre des trinitaires », dans L'École de Saint-Victor de Paris: influence et rayonnement du Moyen Âge à l'époque moderne, éd. Dominique Poirel, Turnhout, Brepols, 2010, p. 459-474, en part. p. 463-464.

26. Festum conceptionis beate Marie Virginis antiquitus erat festum duplex. Sed quia tempore domini Guillelmi abbatis hujus loci fuit magna controversia inter venerabilem universitatem Parisiensem et fratres predicatores seu jacobitas, qui graviter circa dictam conceptionem beate Virginis errabant et gloriosam Virginem in sibi devotis increpabant seu impugnabant; quapropter multi ipsorum fratrum male tractati errores suos publice revocaverunt et, in carceribus positi post multas derisiones quas propter hoc passi sunt, a predicta universitate privati fuerunt; et ideo predictus Aabbas [sic] Guillelmus, tertius sub hoc nomine, motus devotione ad laudem et gloriam benedicte Virginis, statuit et ordinavit istud festum celebrari in parvis capis, et per omnia fieri in talibus festis est fieri consuetum (Paris, BNF, ms. latin 12086, f. 34v-35). 
Trois mots auraient suffi à énoncer le rit in parvis capis affecté à l'office du 8 décembre. Mais sa promotion de fête double à «petites chapes» était trop fraîche pour en avoir oublié la cause. En 1387 éclatait en effet la violente controverse suscitée par Jean de Monzon sur la doctrine de l'Immaculée Conception, et qui valut aux dominicains d'être persécutés, puis exclus de l'Université de Paris deux ans plus tard ${ }^{27}$. Le rédacteur de l'ordinaire de 1395 avait visiblement en mémoire ou sous les yeux le statut tout récent qui modifiait le rit de la fête. La formulation permet d'en reconnaître deux parties: l'exposé, où sont complaisamment relatées les circonstances motivant la décision, et le dispositif, appuyé par la redondance des verbes statuit et ordinavit. Si rares que soient les témoignages de cette sorte, la nature de l'ordinaire comme synthèse des traditions liturgiques promulguée sous l'autorité d'une église ne fait pas de doute. Le prologue de Lisiard, doyen du chapitre de Laon (11551168), introduisant un ordinaire à l'usage de sa cathédrale, a tous les traits d'un statut capitulaire en bonne et due forme, ordonnant la mise par écrit de son usage liturgique (in servitio ecclesie nostre). Sa copie en tête de l'ouvrage réalisé rappelle l'origine du texte et lui donne sa valeur juridique. Par sa rédaction et son vocabulaire, calqués sur ceux d'une charte, il s'apparente aux actes contemporains des évêques de Laon $^{28}$. Ont en effet été conservés la suscription (Ego Lysiardus...), la notification (omnibus...) et le salut (in perpetuum), suivis d'un long préambule (Ea que inter actus humanos...) et du dispositif (communi assensu capituli nostri statutum est...):

Moi Lisiard, doyen de la sainte église-mère de Laon, à tous ceux présents et futurs qui servent fidèlement le Christ, à perpétuité. Les situations que le hasard établit parmi les actes humains sont souvent

27. Voir Marielle Lamy, «Les dominicains dans la tourmente: les suites de l'affaire de Jean de Monzon », dans Religion et société urbaine au Moyen Âge, éd. Patrick Boucheron et Jacques Chiffoleau, Paris, Publications de la Sorbonne, 2000, p. 177-200. Pour plus de détails sur la portée doctrinale de l'affaire, voir Eadem, L'Immaculée Conception. Étapes et enjeux d'une controverse au Moyen Âge (XII'$X V^{e}$ s.), Paris, Institut des Études Augustiniennes, 2000 (Collection des Études Augustiniennes, Série Moyen Âge et Temps Modernes, 35), en part. p. 559-591.

28. Annie Dufour-Malbezin, Actes des évêques de Laon, des origines à 1151, Paris, CNRS, 2001 (Documents, études et répertoires, 65). On relève certains traits communs, comme l'emploi fréquent du pronom ego en tête de la suscription, la formule sancte matris ecclesie Laudunensis (ibid., p. 38), ou encore l'importance donnée dans les préambules aux bienfaits de la «mise par écrit» (ibid., p. 42), thème apparaissant sous l'épiscopat de Barthélemy de Joux (1113-1151). Mais ces caractères ne sont pas exclusifs des chartes laonnoises. 
altérées par le nuage obscurcissant de l'oubli, qui procède de la fragilité du corps humain, et par le passage du cours du temps; aussi faut-il les confier à la cellule de la mémoire pour qu'elles demeurent inchangées ; la raison réclame de les marquer par écrit, c'est ainsi que s'accoutume l'antiquité. Puisque, les personnes succédant les unes aux autres, la dispute survenait très souvent dans nos coutumes, et surtout dans le service de notre église, il a été décidé par commun accord de notre chapitre et approuvé par tous que ce que nous tenons par usage de nos prédécesseurs fût, avec l'aide du conseil divin, mis par écrit, et qu'ainsi confirmé, fût dorénavant tenu pour inébranlable ${ }^{29}$.

Le manuscrit, datant du second quart du XIII ${ }^{\mathrm{e}}$ siècle, est largement postérieur au décanat de Lisiard $^{30}$. L'ordinaire qui suit le prologue correspond-il à la «mise par écrit» de l'usage cathédral annoncée par le doyen du chapitre, ou en est-ce une version ultérieure et déjà remaniée? Aucun indice explicite ne permet de dater le corps du texte. Ce défaut, que partage la majorité des ordinaires médiévaux, explique le silence des sources capitulaires et synodales sur leur promulgation. L'inconvénient de ne pouvoir désigner de manière distinctive les livres liturgiques appartenant à des générations différentes dissuadait fatalement de conserver, voire de consigner les statuts notifiant leur entrée en vigueur. Une solution eût été de mentionner systématiquement, en plus de l'usage, l'année de chaque «édition» dans l'intitulé ou l'incipit du texte, date à laquelle on aurait pu se référer sans équivoque. Mais ce procédé, que la production imprimée implanta grâce aux informations données dans les pages de titre, n'eut pas cours au Moyen $\hat{A}_{g e}{ }^{31}$. Il aurait

29. Ego Lysiardus, sancte matris ecclesie Laudunensis decanus, omnibus tam futuris quam presentibus fideliter Christo servientibus, in perpetuum. Ea que inter actus humanos loca sortiuntur, nubilo oblivionis obfuscante, que ex humani corporis fragilitate progreditur, temporis labente curriculo, multociens alterantur; que vero memoriali cellule committenda sunt, ut immutata permaneant, litteris designari ratio postulat, et sic consuevit antiquitas. Cum igitur in consuetudinibus nostris, diversarum personarum successione, et maxime in servitio ecclesie nostre sepissime altercatio proveniret, communi assensu capituli nostri statutum est et ab omnibus approbatum ut ea que ab antecessoribus nostris usualiter tenemus, divino aminiculante consilio, scripto committerentur, et sic confirmata amodo inconcussa tenerentur. - Laon, BM, ms. 215, f. 43, d'après Ulysse Chevalier, Ordinaires de l'église cathédrale de Laon (XII ${ }^{e}$ et XIII ${ }^{e}$ siècles), Paris, Picard, 1897 (Bibliothèque liturgique, 6), p. 1.

30. Avant 1247, d'après Denis Muzerelle, Geneviève Grand, Guy Lanoë, Olivier Legendre, Dominique Stutzmann, Catalogue des manuscrits datés des Bibliothèques de France. II. Laon, Saint-Quentin, Soissons, à paraître.

31. L'identification du texte par le nom de son auteur aurait pu rendre le même service qu'une mention de date. Mais ce renseignement, quand il est donné, figure à la fin des prologues plutôt qu'à leur début. 
d'ailleurs contrarié une autre des fonctions ces «livres-étalons» d'un usage, à savoir accueillir les strates d'ajouts et de corrections enregistrant les incessants rectificatifs apportés aux célébrations.

\subsection{D'un ordinaire à l'autre}

Il est peu vraisemblable que chacun de ces aménagements ait pu donner lieu à la rédaction d'un nouvel ordinaire. Dans le cadre étroit des liturgies locales, la recomposition fréquente d'un instrument aussi complexe aurait excédé les forces des sacristes. Par chance, les colophons datés de trois ordinaires à l'usage d'Amiens donnent un aperçu du rythme de renouvellement. Le plus ancien est celui élaboré sous la direction de Raoul de Rouvroy pour la cathédrale en 1291 ; le second, de 1306, n'en est que la déclinaison «allégée » à destination des églises paroissiales du diocèse; ce n'est qu'en 1337 que le chanoine Jean Le Moine donna une véritable refonte de la version de $1291^{32}$. Entre ces deux dates, l'exemplaire dû à Raoul de Rouvroy fut remanié, surtout par grattage et réécriture de certains paragraphes. Certes, il ne perdit pas ensuite sa valeur prescriptive, puisqu'il resta enchaîné dans le chœur de la cathédrale jusqu'à l'époque moderne.

Mais cette fonction d'enregistrement des nouvelles coutumes qu'il garda jusqu'à la mise à jour de Jean Le Moine est primordiale. Elle transparaît de façon flagrante dans les cas d'ordinaires jumeaux, comme ceux copiés pour la collégiale Saint-Géry de Cambrai dans le troisième quart du XIII ${ }^{\mathrm{e}}$ siècle: pendant le demi-siècle qui suivit, des corrections similaires furent portées par les mêmes mains sur chacun des deux exemplaires ${ }^{33}$. Cette reportation scrupuleuse manifeste l'autorité dont ils étaient investis conjointement, y compris dans la notification des statuts postérieurs à leur copie primitive. Le fait qu' un amendement fût inscrit sur le ou les «livresétalons» de l'usage lui communiquait donc une sorte de validité juridique, en attendant son éventuelle intégration dans le futur

32. Voir Georges Durand, Ordinaire de l'église Notre-Dame, cathédrale d'Amiens, op. cit., p. IV-VIII, XIII-XIV. Amiens, BM, ms. 184 (ordinaire de 1291); Amiens, AD de la Somme, G 2976 (ordinaire diocésain de 1306); l'ordinaire de Jean Le Moine est principalement connu par les extraits que le chanoine François Villemain en a tiré à la fin du XvII ${ }^{\mathrm{e}}$ siècle (AD de la Somme, G 3027, G 3028). Autres extraits de moindre taille: ibid., G 846 et G 899.

33. Cambrai, BM, ms. 202, et sa copie contemporaine, Cambrai, BM, ms. 44. 
ordinaire recomposé. Cet aspect majeur est trop souvent négligé dans les éditions critiques, qui rejettent en apparat les interventions postérieures à la copie primitive comme des corruptions de l'archétype. Ce choix présuppose la légitimité d'une tradition en vertu de son ancienneté, à rebours du pragmatisme «juridique» qui stimule l'évolution des liturgies. En l'espèce, c'est prendre le contrepied de la mentalité médiévale, qui prise davantage la perpétuation de ses réformes que le souvenir d'usages abrogés.

Documents de la pratique, à la fois codes et registres coutumiers, les ordinaires restaient néanmoins soumis aux contraintes matérielles du support et de la mise en page: impossible d'annoter les marges à l'infini, ou d'éraser sans cesse la copie sans finir par percer le parchemin. Les limites à l'évolution du premier ordinaire à l'usage de l'abbaye Saint-Victor de Paris, datable de la première décennie du XIII ${ }^{\mathrm{e}}$ siècle, furent atteintes bien avant la composition d'une version modernisée, qui eut lieu environ deux cents ans plus tard (peu après 1403), sous l'abbatiat de Geoffroy Pellegay ${ }^{34}$. Dans le dernier tiers du XIII ${ }^{\mathrm{e}}$ siècle, l'empilement en strates successives de notes et de réécritures, elles-mêmes objet de rectifications, empêchèrent de poursuivre la mise à jour systématique de ce document de référence: les systèmes de renvois superposés ne permettaient plus de remédier à l'encombrement des marges sans porter atteinte à la lisibilité du manuscrit. Les victorins, plutôt que de refondre leur vieil ordinaire, préférèrent lui associer un bréviaire $^{35}$. Celui-ci, datable avant 1292, tint lieu de registre des offices institués ou modifiés depuis lors jusqu'à l'établissement d'une nouvelle synthèse sous Geoffroy Pellegay. Un indice permet d'isoler le moment où il commença à prendre le relais de l'ancien volume. Le premier avenant inscrit dans le bréviaire, instituant un jour octave de la Sainte-Marie Madeleine au 29 juillet, a été reporté

34. Paris, BNF, ms. latin 14506, f. 261-333 (ordinaire du début du XIII ${ }^{\mathrm{e}}$ siècle); Paris, BNF, ms. latin 14455 (ordinaire de Geoffroy Pellegay). Voir J.-B. Lebigue, «Le modèle liturgique de Saint-Victor de Paris...», art. cit., p. 466-472. Vers 1240-1250, les trinitaires se servirent du premier ordinaire victorin pour composer une version adaptée à leur usage. Ce fut l'occasion d'intégrer dans le corps du texte les rectifications portées dans le manuscrit depuis sa copie primitive. Mais cette refonte, destinée aux trinitaires, n'était d'aucune utilité pour les chanoines de Saint-Victor.

35. Paris, Bibl. Mazarine, ms. 342 (Bréviaire à l'usage de Saint-Victor de Paris). La séquence chronologique des nouveaux offices adoptés commence à partir du f. 366v. 
par la même main dans l'ordinaire ${ }^{36}$ : cette dernière y ajouta un renvoi (f. 312: require in fine libri) ne pouvant s'appliquer qu'à ce bréviaire précis, où le nouvel office figurait justement à la fin du volume, et non pas à son emplacement logique au milieu du sanctoral.

Les qualités similaires émanant de ces deux types de livres, voués à la consultation et à l'enregistrement de l'usage, obligent à reconsidérer le rôle des bréviaires au Moyen Âge. Il semble qu'une partie d'entre eux n'était pas tant destinée à la récitation effective des heures qu'à servir de guide général aux cérémonies dans la sélection et l'agencement des chants, des oraisons et des lectures de l'office ${ }^{37}$. Cette équivalence relative du rôle de l'ordinaire et du bréviaire recoupe le témoignage des statuts synodaux, qui désignent le livre-étalon d'un usage diocésain soit sous le nom de breviarium, soit sous celui d' ordinarium $^{38}$. Si floue qu' ait pu être la terminologie médiévale des manuscrits liturgiques, son adéquation est ici parfaite avec la réalité des documents.

\subsection{Diffusion des usages}

La diffusion des usages au sein des ordres monastiques, canoniaux ou mendiants a laissé de nombreux vestiges documentaires, tant sous forme d'ordinaires que de statuts généraux, coutumiers, cérémonials, constitutions, etc. La distance, parfois considérable, qui séparait les établissements partageant la même règle de vie rendait nécessaire sa mise par écrit. Toute actualisation passait par la réunion de chapitres généraux, seuls compétents pour sa promulgation et aptes à la propager. Ce principe se vérifie dans la plupart des ordres, même si le tableau doit être nuancé: la cohésion des coutumes liturgiques, très forte chez les cisterciens ou dans les ordres mendiants, est nettement moindre dans les abbayes de chanoines prémontrés ou victorins, qui jouissent ici ou là d'une f. 312 .

36. Paris, Bibl. Mazarine, ms. 342, f. 366v; Paris, BNF, ms. latin 14506,

37. Il est moins nécessaire de pourvoir à l'organisation les messes sur l'année, puisque leur choix est soumis aux règles de préséances régissant l'office. Ce sont aussi ces dernières qui, dans un usage donné, déterminent la distribution éventuelle de propres différents entre les messes conventuelles d'une même journée (messe matutinale et grand messe) et la mémoire des messes empêchées.

38. Voir infra. 
certaine initiative, transparaissant dans l'adoption de fêtes locales ${ }^{39}$. Ces contaminations étaient peut-être imposées par la juridiction épiscopale quand ces maisons n'en étaient pas exemptes, ou qu'elles étaient appelées à participer aux synodes diocésains.

Ces assemblées synodales, dont l'essor date du second tiers du $\mathrm{XII}^{\mathrm{e}}$ siècle, réunissaient annuellement dans la cathédrale le clergé séculier du diocèse autour de l'évêque ${ }^{40}$. Elles furent, concurremment aux chapitres généraux des ordres réguliers, le principal organe de la publication et de la diffusion des usages liturgiques. La plus ancienne collection de statuts synodaux conservée fut élaborée sous la direction de l'évêque de Paris Eudes de Sully, dans la douzaine d'années précédant le quatrième concile général du Latran (1215). Les livres cultuels y font l'objet de deux statuts consécutifs:

Que les prêtres de paroisse aient chacun le livre qu'on appelle manuel, où est contenu l'ordo du service d'extrême onction, celui du catéchisme, du baptême, etc.

Qu'ils aient chacun les canons pénitentiels et l'ordinaire des offices de l'église, selon l'usage et la manière qu'on observe à l'église majeure $^{41}$.

Le «manuel» dont il est question dans le premier paragraphe est l'ancêtre des rituels de l'époque moderne, contenant les prescriptions, enseignements et pièces liturgiques nécessaires à l'administration des sacrements. L'ordinarium officiorum ecclesie mentionné dans le second est un terme générique, dont il est difficile de trancher s'il désigne matériellement un ordinaire ou un bréviaire. Mais il suffit de savoir qu'il pourvoit à l'organisation générale des offices «selon l'usage et la manière qu'on observe à l'église majeure », c'est-à-dire la cathédrale. Les coutumes observées dans «l'église majeure», appelée aussi «église-mère»par d'autres statuts, sont donc la matrice de la liturgie diocésaine: cette réalité est affirmée de plus en plus précisément au cours du XIII ${ }^{\mathrm{e}}$ siècle. Le

39. Voir J.-B. Lebigue, «Le modèle liturgique de Saint-Victor de Paris...», art. cit., p. 462-463.

40. Sur l'origine et les premiers témoins de la législation synodale, voir Odette Pontal, Les statuts synodaux français $d u X^{X I I I^{e}}$ siècle, précédés de l'historique du synode diocésain et de ses origines. T. 1: Les statuts de Paris et le synodal de l'Ouest (XIII siècle), Paris, Bibliothèque nationale, 1971, en part. p. LXXII-LXXVII.

41. Librum qui dicitur manualis habeant singuli sacerdotes parochiales, ubi continetur ordo servitii extreme unctionis, catechismi, baptismi et hujusmodi. / Habeant singuli canones penitentiales et ordinarium officiorum ecclesie, secundum usum et modum qui servatur in majori ecclesia. Ibid., p. 70, $\mathrm{n}^{\text {os }} 49-50$. 
synodal de l'Ouest, légèrement postérieur à Latran IV, mentionne seulement, outre le manuel presbytéral, des «livres dans lesquels on puisse correctement dire l'office divin diurne et nocturne $\gg^{42}$. En 1240 ou 1247, l'évêque du Mans Geoffroy de Loudun le fit remanier et compléta le statut traitant des livres ecclésiastiques par une confirmation explicite du modèle cathédral, diffusé par le biais d'un calendrier et d'un ordinaire:

Item, puisque les membres doivent se conformer à la tête, nous voulons et commandons que chaque prêtre de paroisse ait le calendrier et l'ordinaire du Mans, et qu'il s'applique à observer, dans ses fêtes, ses offices et en tout ce qui par ailleurs lui est possible, l'usage de l'église-mère ${ }^{43}$.

Les collections synodales postérieures réaffirment l'obligation des prêtres de paroisse de se conformer à l'usage de la cathédrale, tel qu'il est marqué dans l'ordinaire ou dans le bréviaire, assortissant d'amendes ou de peines rétorsives tout manquement à son observance ${ }^{44}$. Toutes les cérémonies de la cathédrale n'étaient pourtant pas reproductibles en dehors d'elle, notamment celles requérant le ministère de l'évêque, comme la consécration des saintes huiles le jeudi saint, l'expulsion et la réintégration des pénitents, etc. De même, ne pouvaient convenir aux églises paroissiales les prescriptions des ordinaires décrivant le trajet des processions à travers la ville, les stations dans les églises voisines, la distribution des rôles entre des exécutants appartenant au clergé de la cathédrale ou les expositions de reliques qui y étaient vénérées. Par conséquent, l'ordinaire composé pour l'église-mère excédait leurs besoins. Les bréviaires, cités concurremment aux ordinaires dans les statuts synodaux pour la diffusion de l'usage liturgique, ont certainement tenu un grand rôle dans l'adaptation du modèle cathédral au «service allégé» des paroisses. Mais on voit aussi apparaître, au plus tard au début du XIV siècle, des ordinaires

42. Ibid., p. 154, $\mathrm{n}^{\circ} 25:$ «[...] et libros alios in quibus possit competenter officium tam diurnum quam nocturnum divinum celebrari».

43. Item, cum membra capiti se debeant conformare, volumus et mandamus ut quilibet parochial[e]s sacerdo[te]s calendinarium et ordinarium habeant Cenomanense, et in festis et officiis suis, et in aliis quibus poterunt, usum matris ecclesie studeant observare (D'après Odette Pontal, Les statuts synodaux français..., op. cit. T. 2: Les statuts de 1230 à 1260, Paris, CTHS, 1983, p. 159, $\mathrm{n}^{\mathrm{o}} 63$ ).

44. Voir ibid., p. 130, $\mathrm{n}^{\circ} 3$ (Rouen); Joseph Avril, Les statuts synodaux français..., op. cit. T. 4: Les statuts synodaux de l'ancienne province de Reims, Paris, CTHS, 1995, p. 251, nº 91 (Noyon). 
dédoublés pour le même usage: l'un, plénier, destiné à l'église-mère, l'autre, sa version réduite pour répondre aux besoins des prêtres diocésains. A déjà été évoqué plus haut le cas d'Amiens, avec la réplique réduite de l'ordinaire de Raoul de Rouvroy, composée en 1306. Le P. Pierre-Marie Gy a signalé un fait similaire pour l'église de Mende à la même époque ${ }^{45}$. Mais l'exemple le plus intéressant a été découvert par Olivier Legendre par comparaison de deux ordinaires contemporains ( $2^{\mathrm{e}}$ quart ou milieu du XIV ${ }^{\mathrm{e}}$ siècle), l'un à l'usage de la cathédrale de Troyes, l'autre à celui des paroisses. Il a démontré que le second n'est pas une réduction expurgée du premier, mais que les deux exemplaires ont été conçus ensemble, sans doute en commençant par la version diocésaine: certaines prescriptions, ne pouvant concerner que les prêtres de paroisses, ont en effet été maintenues par erreur dans l'ordinaire de la cathédrale ${ }^{46}$.

Ce dédoublement des ordinaires séculiers fait du synode l'organe naturel de leur promulgation. Néanmoins, le lieu de leur conception resta sans doute le chapitre de la cathédrale, où se trouvaient à la fois les documents de référence et les spécialistes capables de composer de pareilles synthèses ${ }^{47}$. Plus difficile à évaluer est le degré d'intervention de l'évêque dans la liturgie de son église: ce n'est pas parce que son nom est attaché à la promulgation d'un statut qu'il en est l'auteur ou qu' on lui laisse toute latitude dans la réforme de l'usage. À Amiens, le serment qu'il prêtait sur les évangiles devant les chanoines l'engageait à «conserver perpétuellement, conformément aux institutions canoniques et sauf son droit et son ordre, les droits de l'Église d'Amiens et ses coutumes antiques et approuvées par ses prédécesseurs évêques d'Amiens $»^{48}$. Il est

45. Pierre-Marie Gy, «L'ordinaire de Mende, une œuvre inédite de Guillaume Durand l'Ancien », dans Liturgie et musique (IX ${ }^{e}-X I V^{e}$ s.), Toulouse-Fanjeaux, 1982 (Cahiers de Fanjeaux, 17), p. 239-249: Paris, Bibl. de l'École des Beaux-Arts, Collection Masson, ms. 3 (ordinaire à l'usage de la cathédrale de Mende); Mende, $\mathrm{BM}, \mathrm{ms} .2$ (ordinaire diocésain de Mende).

46. Troyes, BM, ms. 833 (ordinaire à l'usage de la cathédrale de Troyes); Troyes, BM, ms. 1630 (ordinaire diocésain de Troyes). Cas présenté à l'occasion d'une séance du séminaire «L'Église et les églises en Occident au Moyen Âge», 5 octobre 2007: Olivier Legendre et J.-B. Lebigue, «Préserver, unifier? À quoi sert un ordinaire liturgique?»

47. Les auteurs connus d'ordinaires ne sont pas toujours le chantre ou le préchantre du chapitre, même si leurs fonctions les y prédisposent.

48. Traduction d'après Amiens, BM, ms. 388, f. 51. Formule de serment contenue dans les statuts capitulaires d'Amiens, compilés par Pierre Alays, chantre de la cathédrale, en 1412: «Ego N., Ambianensis episcopus, jura et consuetudines 
d'autant plus probable que l'initiative en matière liturgique soit principalement restée aux chapitres, que leur participation active est attestée dans la préparation d'autres statuts synodaux ${ }^{49}$.

Il faudrait aussi cerner l'influence de la liturgie de la cathédrale sur les autres institutions ecclésiastiques représentées au synode, notamment les collégiales. Parmi les très rares exemples de statuts subsistants à régler un aménagement cultuel précis, on relève celui de 1314 à Angers, où l'évêque Guillaume Le Maire institue les octaves de la Toussaint et réforme en conséquence le programme des lectures bibliques du mois de novembre ${ }^{50}$. Cette promulgation synodale, exceptionnellement développée, est assortie d'une annexe qui a toute les caractéristiques d'un article d'ordinaire (prescriptions et incipit de pièces liturgiques compilés dans l'ordre des cérémonies). Or ce statut a été résumé et intégré dans un ordinaire à l'usage de la collégiale Saint-Pierre d'Angers ( $2^{\mathrm{e}}$ quart ou milieu du XIV $\mathrm{XV}^{\mathrm{e}}$ siècle), avec une référence explicite à «cette ordonnance prononcée par Guillaume évêque d'Angers en plein synode de la Saint-Luc évangéliste [18 octobre], l'an du Seigneur $1314 »^{51}$. Comment interpréter cette précision insolite? Témoigne-t-elle d'une procédure courante, ou est-elle justement mentionnée parce qu'étrangère aux habitudes du chapitre de la collégiale dans l'aménagement de sa liturgie? Sans doute ne faut-il pas sous-estimer la variété des situations, ni le poids institutionnel, selon que les collèges soient ou non composés de conchanoines de la cathédrale.

Reste que le tour de plus en plus juridique que prit la promulgation des usages depuis le XIII ${ }^{\mathrm{e}}$ siècle est partout sensible. Les modèles centralisés offerts par certains ordres comme les cisterciens ont pu inspirer ce changement de nature. L'empirisme qui laissait

ecclesie Ambianensis antiquas et approbatas et a predecessoribus meis Ambianensis episcopis hactenus observatas, salvo jure et ordine meo, prepositis sacro sanctis euvangeliis secundum canonica instituta perpetua me servaturum promitto. »

49. Voir Odette Pontal, Les statuts synodaux français... T. 1: Les statuts de Paris..., op. cit., p. LXIV.

50. Voir Joseph Avril, Les statuts synodaux français..., op. cit. T. 3: Les statuts synodaux angevins de la seconde moitié du XIII siècle, précédés d'une étude sur la législation synodale angevine, Paris, CTHS, 1988, p. 234-242.

51. Cité ibid., p. 238-239, n. 10. 
aux chapitres une relative indépendance dans la perpétuation et l'enregistrement de leurs coutumes liturgiques fut contrarié par l'essor de la législation synodale, organe de diffusion parfaitement adapté, mais suscitant l'intervention de l'évêque ${ }^{52}$. Les chanoines gardèrent longtemps la main sur l'usage, étant seuls capables de fournir des synthèses aussi complexes que les ordinaires. Jusqu'au début de l'époque moderne, les chapitres de cathédrales purent se targuer d'être les dépositaires des traditions. C'est dans cet esprit que les chanoines faisaient encore copier et enluminer au début $\mathrm{du} \mathrm{XVI} \mathrm{I}^{\mathrm{e}}$ siècle des manuscrits pontificaux conformes à l'usage de l'église locale pour les offrir à leur nouvel évêque: les écus laissés en réserve dans les marges n'étaient peints aux armes du prélat qu'au moment de son élection ${ }^{53}$. Les chapitres luttaient ainsi contre le mouvement d'uniformisation sur l'usage de la curie romaine, dont les indices, encore discrets au Moyen Âge, pouvaient à juste titre les inquiéter: l'incoercible succès du pontifical de Guillaume Durand aux dépens des recueils locaux, celui de son Rational des divins offices, où les diverses pratiques cultuelles étaient rapportées à l'aune de la liturgie romaine; mais aussi l'interférence de la juridiction papale dans l'institution de fêtes «universelles», liées à la confiscation à son profit des procédures de canonisation des saints, sans oublier la propagation souterraine de l'usage romain par les frères mineurs et la dévotion d'inspiration franciscaine.

$\mathrm{Ce}$ mouvement alla s'aggravant au $\mathrm{XVI}^{\mathrm{e}}$ siècle. L'imprimerie en pleine expansion favorisa inéluctablement les livres de la liturgie romaine, même si les églises locales eurent aussi leurs propres productions, concurremment à la copie manuscrite ${ }^{54}$.

52. Rappelons que l'évêque n'était pas toujours, loin s'en faut, membre du chapitre de sa cathédrale.

53. Par exemple un pontifical à l'usage d'Amiens (Besançon, BM, ms. 135), aux armes de François Halwin, élu en 1504; ou le recueil pontifical de cathédrale à l'usage d'Évreux (Paris, BNF, ms. latin 963), dans les marges duquel on reconnaît le blason de Raoul du Fou (1479-1511), ajouté peu après l'enluminure primitive. Voir J.-B. Lebigue, «Chapelles livresques et ministres désignés...», art. cit., p. 26-28.

54. Voir le cas lyonnais exploré par Robert Amiet, Inventaire général des livres liturgiques du diocèse de Lyon, Paris, CNRS, 1979. Sauf quand les œuvres d'enlumineurs tardifs ou de compositeurs de musique sacrée ont attiré l'attention d'historiens de l'art et de musicologues, les manuscrits liturgiques de l'époque moderne restent à ce jour un champ vierge de recherche. Écartés des catalogues conçus par des médiévistes, dédaignés des modernistes plus intéressés par la production imprimée, ils constituent pourtant une part non négligeable des manuscrits cultuels conservés dans les bibliothèques. Quelques exemples 
L'esprit humaniste, avide d'épurer l'héritage antique de ses scories médiévales, porta un coup bien plus rude à la législation des usages. À prôner le retour, du reste illusoire, à la liturgie de l'Église des Pères, il assimilait les particularismes cultuels à la corruption d'une tradition préexistante, laquelle se serait maintenue à Rome plus fidèlement qu'ailleurs. Les sacristes recherchèrent dès lors un archétype de la liturgie romaine dans les témoins manuscrits les plus anciens, leur transfusant de fait l'autorité qu'ils niaient aux institutions médiévales postérieures ${ }^{55}$. C'est dans ce but avoué de rétablir la «forme primitive» de l'office divin qu'un franciscain, le cardinal François Quignonez, publia un Breviarium romanum en $1535^{56}$. Mal conçu, d'où son maigre succès, l'ouvrage fondait néanmoins dans ses principes la réforme du Bréviaire et du Missel que les Pères du concile de Trente confièrent au pape Pie IV en 1563: les éditions révisées en conséquence furent promulguées par Pie V, respectivement en 1568 et 1570 . L'adoption massive de l'usage romain «restauré» peut surprendre. Le décret dont elle était assortie ne la rendait obligatoire que pour les églises ne pouvant justifier d'un rite attesté depuis plus de deux siècles: or les preuves ne manquaient pas dans la plupart des chapitres et ordres. Seul permet de comprendre une telle abdication le traumatisme des Guerres de religion, qui ruinèrent en grand nombre les temples catholiques, sans excepter les livres liturgiques placés dans les chœurs, trésors et sacristies ${ }^{57}$. Ces dévastations combinées à l'esprit du temps, affaiblissant les réflexes identitaires des églises, rencontrèrent sans doute les préoccupations des évêques, soucieux

sont décrits dans J.-B. Lebigue, Catalogue de manuscrits liturgiques médiévaux et modernes, Orléans, IRHT, 2006-2010 (Ædilis, Publications scientifiques, 7). En ligne: http://www.cn-telma.fr/liturgie/.

55. Ce parti pris a longtemps faussé l'analyse des documents liturgiques hérités du Haut Moyen Âge, trop souvent interprétés comme des modèles de romanité, en dépit de leur composante franco-gallicane. Depuis quelques années, ce préjugé historiographique, qu'avait renforcé le puissant courant grégorianiste des $\mathrm{XIX}^{\mathrm{e}}$ et $\mathrm{XX}^{\mathrm{e}}$ siècles, est vigoureusement attaqué entre autres par Philippe Bernard, Transitions liturgiques en Gaule carolingienne: une traduction commentée des deux lettres faussement attribuées à l'évêque Germain de Paris (fin du vIII siècle), Paris, Hora decima, 2008.

56. Voir Pierre Salmon, L'office divin au Moyen Âge, Paris, Cerf, 1967 (Lex orandi, 43), en part. p. 178-184, en faisant abstraction du «déterminisme romain» qui ressort de son propos.

57. Voir Mathieu Lours, L'autre temps des cathédrales, du concile de Trente à la Révolution française, Paris, Picard, 2010, en part. p. 90-93. 
de ressaisir le gouvernement effectif du culte dans leurs diocèses. Il était en effet tentant de substituer aux coutumes locales, difficiles à maîtriser, un modèle romain unifié.

L'abandon de la majorité des usages du Moyen Âge à l'occasion de la réforme promulguée par Pie $\mathrm{V}$, renforcée par la création de la Sacrée Congrégation des Rites en 1588, marqua ainsi la défaite irrémédiable des chapitres cathédraux. La dépossession dont ils furent victimes en perdant la capacité d'édicter les coutumes liturgiques les condamnait à vivre en marge du modèle pastoral issu du concile de Trente, centré sur l'évêque et le prêtre de paroisse. Elle explique enfin la disparition brutale des ordinaires au $\mathrm{XVI}^{\mathrm{e}}$ siècle, devenus obsolètes dans leur contenu comme dans leur principe. Jusqu'à la fin de l'Ancien Régime, les chanoines continuèrent à s'y référer, voire à les annoter; mais ils le firent non plus en vue de promulguer les rectifications de leur usage, mais comme on consulte, en érudit, le témoin d'une antiquité vénérable.

lebigue@cnrs-orleans.fr 